(1)

CrossMark

\title{
A crossroads between the heart and lungs: air pollution and pulmonary hypertension
}

\author{
Meredith C. McCormack and Stephen C. Mathai \\ Affiliation: Pulmonary and Critical Care Medicine, Johns Hopkins University, Baltimore, MD, USA.
}

Correspondence: Meredith C. McCormack, Pulmonary and Critical Care Medicine, Johns Hopkins University, 1830 E Monument St, 5th Floor, Baltimore, MD 21205, USA. E-mail: mmccor16ajhmi.edu

@ERSpublications

Proximity to traffic and particulate pollution may be associated with disease severity and increased mortality in individuals with pulmonary arterial hypertension. Future studies are needed to confirm these findings. http://bit.ly/2P5QE52

Cite this article as: McCormack MC, Mathai SC. A crossroads between the heart and lungs: air pollution and pulmonary hypertension. Eur Respir J 2019; 53: 1900654 [https://doi.org/10.1183/13993003.006542019].

While most studies of air pollution health effects have been in the general population, studying subgroups with chronic disease has the potential to define the magnitude of the health effects in sensitive populations and may provide unique insights into the mechanism of pollutant effects. Studies have shown indoor air pollution to be associated with increased chronic obstructive pulmonary disease (COPD) morbidity [1] and outdoor air pollution with increased COPD morbidity and mortality [2]. Recent work in idiopathic pulmonary fibrosis (IPF) has suggested that outdoor air pollution may be associated with steeper rate of lung function decline, risk of IPF exacerbation, and increase in mortality [3-5]. While these and other studies have investigated lung function, exacerbations and mortality, air pollution studies have not previously included assessment of pulmonary vascular effects, as this outcome is not easily accessible. Pulmonary arterial hypertension (PAH) cohorts often include catherisation data as part of longitudinal assessment of disease course and thus, studies in populations with pulmonary hypertension provide a unique opportunity to link air pollution exposure to physiological effects on the pulmonary circulation.

In this issue of the European Respiratory Journal, Sofianopoulou et al. [6] report findings from the UK National Cohort Study linking traffic-related pollution to health outcomes among those with underlying $\mathrm{PAH}$. The cohort of $\sim 300$ individuals, while relatively small, was well characterised clinically and had right heart catherisation measurements, providing the ability to investigate associations with physiological end-points. The investigators applied geocoding to assign annual average exposure to particulate matter with a $50 \%$ cut-off aerodynamic diameter $\leqslant 2.5 \mu \mathrm{m}(\mathrm{PM} 2.5)$ and $\mathrm{NO}_{2}$ based on 2010 concentrations, which preceded participant entry into the cohort and were selected as indicators of chronic exposure. Proximity of the home residence to roadway and length of roads near the home were also used to represent traffic exposure.

Proximity to traffic was associated with worse disease severity as assessed by cardiopulmonary haemodynamics and by the European Respiratory Society and European Society of Cardiology PAH risk score [7]. However, proximity to traffic was not associated with transplant-free survival. Conversely, chronic PM2.5 was associated with reduced transplant-free survival. The magnitude of effect of PM2.5 on outcomes was greater than most prior studies with a more than two-fold increased risk of transplant or 
death (HR 2.47, 95\% CI 1.12-5.48) per $3 \mu \mathrm{g} \cdot \mathrm{m}^{-3}$ increase in PM2.5. This was in the absence of extraordinarily high particulate matter exposure. In fact, the PM2.5 concentration was generally within regulatory agency recommended ranges, with an ambient PM2.5 concentration median value of $13.7 \mathrm{ug} \cdot \mathrm{m}^{-3}$. Unexpectedly, PM2.5 exposure was associated with lower pulmonary vascular resistance (PVR). This was surprising as higher PVR predicts poorer survival in PAH and thus, one would expect an association between long-term PM2.5 exposure and mortality in PAH to be reflected by similar changes in pulmonary haemodynamics. It is possible that the observed association between PM2.5 and outcomes may not be mediated through PVR or that PVR is an inadequate tool to assess this relationship, particularly if the effects of PM2.5 are directed at the right ventricle (RV) rather than the pulmonary vasculature. AARON et al. [8] have demonstrated an association between PM2.5 exposure and RV structure and function as assessed by cardiac magnetic resonance imaging (CMR) in the Multi-Ethnic Study of Atherosclerosis (MESA), demonstrating increasing RV mass with increasing particulate matter exposure in healthy adults. Furthermore, prior work in a cohort of PAH demonstrated that more than $25 \%$ of patients who experienced improvement in PVR with pulmonary vasodilator therapy had a concomitant decline in RV function as assessed by CMR, suggesting that PVR may be an inadequate measure of RV function [9]. Since PM2.5 may have direct effects on the RV, and RV function is the main determinant of outcomes in $\mathrm{PAH}$, better characterisation of the relationship is needed.

Interestingly, the investigators found no association between $\mathrm{NO}_{2}$ exposure and disease severity or transplant-free survival. This too was surprising, given prior research demonstrating a strong relationship between $\mathrm{NO}_{2}$ and $\mathrm{RV}$ structure and function. In a separate study from $\mathrm{MESA}, \mathrm{NO}_{2}$ was used as a surrogate for traffic exposure and was associated with increases in RV mass and in end diastolic volume among over 4000 participants [10]. The MESA investigators speculated that traffic-related pollution leads to increases in pulmonary vascular resistance and right heart hypertrophy. While MESA had the advantage of CMR, catherisation data was not available and the results supported the need to study the effects of pollution on the pulmonary vasculature in potentially susceptible populations [11].

The work of Sofianopoulou et al. [6] extends the robust literature demonstrating the association between air pollution and cardiopulmonary health effects in general and susceptible populations [12-14]. Recent evidence from the MESA study has advanced our understanding of air pollution health effects by reporting associations between air pollution exposure and right heart structure and function in the general population $[8,10,15]$. This has led to questions about the potential effect of air pollution on the pulmonary vasculature. The concept that air pollution may provoke changes in the pulmonary vasculature that lead to pulmonary hypertension has biological plausibility given the proximity of pulmonary vasculature to the alveolar-capillary interface and the potential for pollution to either act directly or through translocation into the circulation to elicit inflammation, oxidative stress and coagulation responses. Evidence from animal models suggests that air pollution exposure, including particulate matter and diesel exhaust, results in changes to the pulmonary vasculature $[16,17]$. Despite some limitations of the study design, the findings from the UK cohort study represent an important step in addressing gaps in the current understanding of air pollution health effects on the pulmonary vasculature.

Lack of consistency in associations between 1) traffic exposure versus PM2.5 and disease severity and 2) the results related to $\mathrm{NO}_{2}$ exposure and prior studies suggest the study results need to be interpreted with caution. These discrepancies could be an indicator of exposure misclassification, unmeasured confounders, or could represent heterogeneity of effects of the multiple exposures represented by traffic. Individuals spend the majority of their time indoors and those with chronic diseases are even more likely to remain in their homes. Indoor air pollution and other home environmental exposures may be drivers of health effects and these were not measured as part of this study. It is also possible that there was variability in the time of clinical presentation that was driven by participant factors and that this influenced the cross-sectional analysis between air pollution and baseline haemodynamics.

Sofianopoulou et al. [6] should be congratulated for their novel application of data from the UK cohort study to address the question of pollutant effects in PAH. Their study suggests that PAH may be a promising model with which to study the effects of air pollution on the pulmonary vasculature. Future studies may include more detailed assessment of indoor and outdoor pollutant exposure and longitudinal design to define the effect of pollution exposure on changes in pulmonary vasculature and right heart over time. While PAH is a relatively rare disease which creates challenges in achieving larger sample sizes, the existence of cohorts that include biological and physiological indicators of disease provides a unique opportunity. The biological rationale that patients with $\mathrm{PAH}$ may represent a susceptible subgroup is compelling and the investigators build a strong case for the need for future studies to replicate their findings and advance our understanding on pollution health effects on the pulmonary vasculature in general and in potentially uniquely susceptible patients with $\mathrm{PAH}$. 
Conflict of interest: M.C. McCormack reports authorship royalties from UptoDate, outside the submitted work. S.C. Mathai reports personal fees for consultancy from Arena and Actelion, personal fees for consultancy and data monitoring committee work from United Therapeutics, during the conduct of the study; and is a member of the Scientific Leadership Council, Pulmonary Hypertension Association.

\section{References}

1 Hansel NN, McCormack MC, Diette GD, et al. In-home air pollution is linked to respiratory morbidity in former smokers with COPD. Am J Respir Crit Care Med 2013; 187: 1098-1103.

2 Gan WQ, FitzGerald JM, Carlsten C, et al. Associations of ambient air pollution with chronic obstructive pulmonary disease hospitalization and mortality. Am J Respir Crit Care Med 2013; 187: 721-727.

3 Johannson KA, Vittinghoff E, Lee K, et al. Acute exacerbation of idiopathic pulmonary fibrosis associated with air pollution exposure. Eur Respir J 2014; 43: 1124-1131.

4 Johannson KA, Vittinghoff E, Morisset J, et al. Air pollution exposure is associated with lower lung function, but not changes in lung function, in patients with idiopathic pulmonary fibrosis. Chest 2018; 154: 119-125.

5 Sese L, Nunes H, Cottin V, et al. Role of atmospheric pollution on the natural history of idiopathic pulmonary fibrosis. Thorax 2018; 73: 145-150.

6 Sofianopoulou E, Kaptoge S, Gräf S. Traffic exposures, air pollution and outcomes in pulmonary arteria hypertension: a UK cohort study analysis. Eur Respir J 2019; 53: 1801429.

7 Galie N, Humbert M, Vachiery JL, et al. 2015 ESC/ERS Guidelines for the diagnosis and treatment of pulmonary hypertension: The Joint Task Force for the Diagnosis and Treatment of Pulmonary Hypertension of the European Society of Cardiology (ESC) and the European Respiratory Society (ERS). Eur Respir J 2015; 46: 903-975.

8 Aaron CP, Chervona Y, Kawut SM, et al. Particulate matter exposure and cardiopulmonary differences in the Multi-Ethnic Study of Atherosclerosis. Environ Health Perspect 2016; 124: 1166-1173.

9 van de Veerdonk MC, Kind T, Marcus JT, et al. Progressive right ventricular dysfunction in patients with pulmonary arterial hypertension responding to therapy. J Am Coll Cardiol 2011; 58: 2511-2519.

10 Leary PJ, Kaufman JD, Barr RG, et al. Traffic-related air pollution and the right ventricle. The Multi-ethnic Study of Atherosclerosis. Am J Respir Crit Care Med 2014; 189: 1093-1100.

11 Holguin F, McCormack MC. Right from wrong: the effect of traffic-related pollution on the right heart. Am J Respir Crit Care Med 2014; 189: 1018-1019.

12 Brook RD, Rajagopalan S, Pope CA III, et al. Particulate matter air pollution and cardiovascular disease: an update to the scientific statement from the American Heart Association. Circulation 2010; 121: 2331-2378.

13 Shah AS, Langrish JP, Nair H, et al. Global association of air pollution and heart failure: a systematic review and meta-analysis. Lancet 2013; 382: 1039-1048.

14 Hansel NN, McCormack MN, Kim V. The effects of air pollution and temperature on COPD. COPD 2016; 13: 372-379.

15 D'Souza JC, Kawut SM, Elkayam LR, et al. Ambient coarse particulate matter and the right ventricle: the Multi-Ethnic Study of Atherosclerosis. Environ Health Perspect 2017; 125: 077019.

16 Park SH, Chen WC, Durmus N, et al. the effects of antigen-specific IgG1 antibody for the pulmonaryhypertension-phenotype and B cells for inflammation in mice exposed to antigen and fine particles from air pollution. PloS One 2015; 10: e0129910.

$17 \mathrm{Lu} \mathrm{J}$, Ye X, Ji D, et al. Diesel exhaust inhalation exposure induces pulmonary arterial hypertension in mice. Environ Pollut 2018; 237: 747-755. 